\title{
A Post-PARTUM Woman WiTH Dyspnea
}

\author{
Saaron Laighold, $M D$
}

A G1P1 40 year-old Caucasian female without significant past medical history presented to an outside hospital with worsening shortness of breath and hemoptysis. The patient was 10 days status post caesarian section for preeclampsia. Her course after delivery was complicated by a stay in the ICU for shortness of breath. She was discharged home 7 days post-delivery and then returned to the hospital 3 days later. The patient was intubated at the outside hospital for hypoxia (O2 sats of $50 \%$ on room air) and transferred to TJUH for further management.

The patient's family history was significant for her father being deceased at age 40 from an MI and her mother being alive and well. The patient lives at home with her husband and her child who is healthy and well. She has no history of drug, alcohol, or cigarette smoking.

Prior to delivery her medications included multivitamin, folate, and ferrous sulfate. After delivery, the patient was discharged home on metoprolol extended release $25 \mathrm{mg}$, furosemide, and hydralazine. Upon transfer to TJUH she was on a dobutamine drip, propofol drip, nitroglycerin drip, and intravenous enalapril.

The patient was transferred to the TJUH CCU already intubated and sedated. Vitals signs were stable with temperature of $97.8 \mathrm{~F}$, pulse of 105, respiratory rate of 19 and blood pressure of 143/78. The physical exam was significant for jugular venous distension to $15 \mathrm{~cm}$, presence of a third heart sound (S3), soft systolic ejection murmur, bilateral coarse breath sounds, and $2+$ lower extremity edema to her knees.

Laboratory values including CBC, chemistry panel, cardiac enzymes and electrolytes were normal at time of transfer. BNP was 158 , protein was $4.7 \mathrm{~g} / \mathrm{L}$, and albumin $2.9 \mathrm{~g} / \mathrm{L}$. Urinalysis revealed $3+$ blood and trace ketones while serum cortisol was 17.5 and TSH was $1.95 \mathrm{mIU} / \mathrm{mL}$.

Studies at the outside hospital showed no pulmonary embolus on CT scan of the chest and no deep vein thrombosis by lower extremity dopplers. Echocardiogram revealed left ventricular ejection fraction of $25-30 \%$ and moderate to severe mitral regurgitation. Chest $\mathrm{x}$-ray on arrival to TJUH was consistent with pulmonary edema as shown below (Figure 1).

Differential diagnosis included peripartum cardiomyopathy, accelerated hypertension, diastolic dysfunction, systemic infection, pulmonary embolism, preeclampsia, amniotic fluid embolus, or cardiomyopathy due to infection; ischemia; or toxicity. The time course, clinical presentation, and studies helped to narrow in on the etiology of her symptoms.

The patient presented 10 days post-partum with signs of left-sided congestive heart failure with a decreased ejection fraction on her echocardiogram. The fact that the patient presented in the postpartum period rather than earlier in her pregnancy would seem to eliminate an underlying cardiomyopathy. Her echocardiogram revealed systolic dysfunction making diastolic dysfunction unlikely to be the cause for this woman's symptoms. She was ruled out for a pulmonary embolus and did not have any signs of systemic infections or indications of an underlying ischemia to account for the new onset cardiomyopathy.

Amniotic fluid embolism can cause hypoxia in the post-partum period. However, the onset of symptoms typically presents during labor and delivery or within the first 48 hours after delivery as opposed to symptoms of severe hypoxia 10 days after delivery, as was the case with this patient. Also, patients with amniotic fluid embolism will typically present with disseminated intravascular coagulation and symptoms similar patients with anaphylactic or septic shock.

Preeclampsia and accelerated hypertension were though to be implausible causes in this case because the patient did not present with severe hypertension at the time of her severe hypoxia. The patient's symptoms of preeclampsia should have improved after delivery instead of causing her to become hypoxic 10 days post delivery.

The patient's presentation was thought to be consistent with peripartum cardiomyopathy. She was treated for congestive heart failure with intravenous furosemide and ACE inhibitor along with the dobutamine and nitrates. After several days of diuresis, a repeat echocardiogram revealed mild to moderate mitral regurgitation, moderate left atrium enlargement, and an ejection fraction of 40$45 \%$ with mild global LV dysfunction. The patient was extubated after several days and transferred to a telemetry floor. She continued to improve with medical treatment for her heart failure. The patient was discharged home on an ACE inhibitor, betablocker and diuretics with the recommendation that she avoid future pregnancies.

The patient had several risk factors for the condition including older maternal age and the development of preeclampsia during her pregnancy. The improvement in symptoms and the improvement in LVEF with supportive therapy were consistent with a peripartum cardiomyopathy as opposed to other causes for her presentation.

\section{Discussion}

Peripartum cardiomyopathy (PPCM) was first described in the 1700 s and first defined in 1937. It is a rare form of dilated cardiomyopathy of unknown etiology which affects women in late pregnancy or early post-partum. The true incidence of this condition is unknown but it is thought to be 1 per 3000 to 1 per 4000 live births or between 1000 and 1300 women each year in the US. For unknown reasons, the incidence of PPCM is higher in certain regions of the world. In one area of Haiti the incidence is estimated to be 1 in 300 pregnancies. 


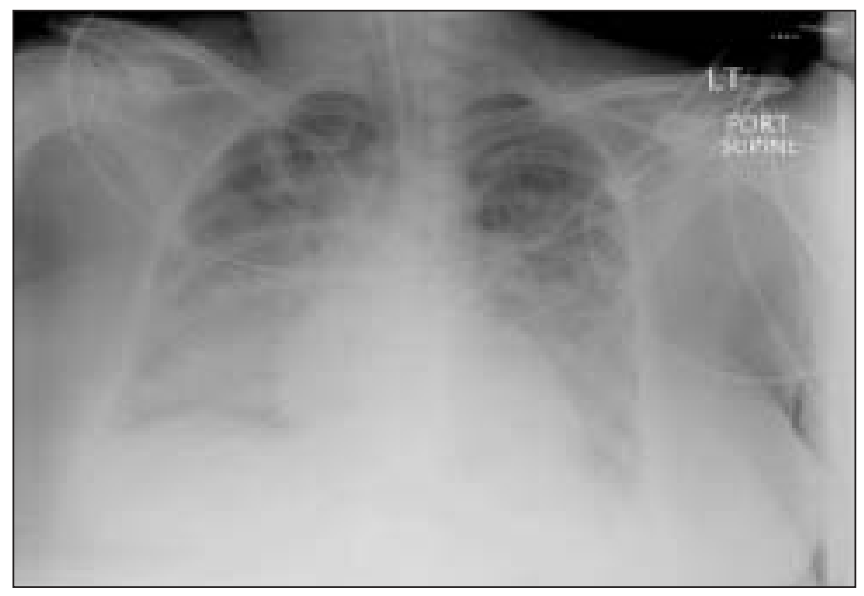

Figure 1. CXR showing pulmonary edema.

Peripartum cardiomyopathy is defined by cardiac failure within the last month of pregnancy or within 5 months postpartum. The condition is characterized by the absence of other causes of congestive heart failure and the lack of heart disease prior to the last month of pregnancy. Most patients with PPCM present with symptoms of congestive heart failure within the first 4 months post-partum (78\%). $9 \%$ of patients present in the last month before delivery while $13 \%$ present more than 1 month antepartum or more than 4 months postpartum. The time course for peripartum cardiomyopathy contrasts with the time for presentation of congestive heart failure due to other etiologies. The 2 nd trimester of pregnancy is the greatest hemodynamic burden of gravid state and most patients with underlying cardiac dysfunction tend to develop symptoms of heart failure at this time. Normal pregnancy causes increased blood volume, increased cardiac output and decreased afterload so that patients with underlying cardiac disease present earlier during pregnancy than patients with PPCM.

Patients are judged to have PPCM after they present in congestive heart failure within the last month of pregnancy or within the first 5 months postpartum. By echo criteria, women with PPCM have $\mathrm{LVEF}<45 \%$, decreased fractional shortening $<30 \%$, and end/diastolic dimension $>2.7 \mathrm{~cm} / \mathrm{m} 3$. Symptoms for congestive heart failure in patients with peripartum cardiomyopathy are similar to heart failure symptoms for patients with other etiologies of CHF. Symptoms include dyspnea, orthopnea, PND, MR murmur. Physical exam signs include cardiomegaly, S3, JVD, peripepheral edema, arrhythmias, and pulmonary rales.

While the cause of peripartum cardiomyopathy remains unknown a number of risk factors are associated with the development of the condition. Risk factors include age greater than 30 years, multiparity, women of African descent, pregnancy with multiple fetuses, history of preeclampsia, eclampsia, postpartum HTN, cocaine abese, nutritional deficiencies including Selenium, and long term oral tocolytic therapy with Beta-agonists such as terbutaline.

While the etiology of PPCM remains elusive, investigation of women with PPCM and associated risk factors has led to identification of several possible etiologies. Viral myocarditis is one suspected cause of PPCM. Studies have reported the incidence of myocarditis among PPCM patients to be from $9 \%$ to $78 \%$. Some studies have estimated the incidence of myocarditis to be similar to controls with idiopathic dilated cardiomyopathy while other studies have estimated the incidence of myocarditis to be much higher among patients with PPCM. The variation among study results is thought to be due differences in the timing of myocardial biopsies and due to variations in the definition of myocarditis used by pathologists. Investigators have speculated that pregnancy may increase patient susceptibility to viral myocarditis leading to PPCM.

It has been postulated that PPCM may be caused by an autoimmune reaction. Supporting evidence for this theory includes the finding that high titers of autoantibodies against cardiac tissue proteins have been identified in women with PPCM. The suspected autoimmune mechanism is that fetal cells escape into the maternal circulation and take up residence in cardiac tissue triggering an autoimmune reaction against the myocardium. After delivery, it is theorized, the fetal cells in the myocardium are recognized as nonself when the autoimmunosuppresion of pregnancy subsides and the patient's immune system attacks the cardiac myocytes causing cardiomyopathy.

Other theories for the etiology of PPCM include systemic inflammation, hormonal involvement and nutritional deficiencies. Inflammation is believed to be involved in the pathogenesis of PPCM as it does in other etiologies of CHF. TNF-alpha, IL-6, Fas/APO-1 are significantly elevated in patients with PPCM compared to controls. FAS: apoptosis-signaling receptor is significantly elevated in patients who died from PPCM as compared to controls. Relaxin is an ovarian hormone produced during pregnancy which is thought to play a role in PPCM. Relaxin has positive inotropic and chonotropic properties and abnormalities of this hormone are speculated to participate in the pathogenesis of PPCM. Nutritional deficiencies, especially selenium deficiency have been found among women with PPCM. In one area of Haiti where PPCM is exceptionally common pregnant women commonly suffer from nutritional deficiencies. Selenium deficiency may play a role in the pathogenesis of PPCM because it may make the heart more susceptible to injury from viruses, hypertension, or hypocalcemia.

Therapy for PPCM involves standard heart failure management including diuretics, inotropes, and blood pressure control. Management of PPCM in the antepartum period must account for possible teratogenecity of certain medications typically used for heart failure patients. ACE inhibitors are contraindicated in 
pregnancy and so they should be avoided. Hydralazine and nitrates are safer to use for blood pressure therapy. Similarly, anticoagulation to prevent the risk of embolism formation should avoid coumadin in the antepartum period because of its possible teratogenecity. Both heparin and coumadin should be safe to use postpartum because neither are excreted into breast milk. If the diagnosis of PPCM is made in the antepartum period, delivery should be strongly considered because of the risk of continuing the pregnancy to both the fetus and the mother.

PPCM has a high incidence of serious morbidity and mortality with listed mortality rates between $18 \%$ and $56 \%$. Complications from PPCM include arrhythmias, progressive heart failure necessitating $\mathrm{BiV}$ pacemaker and defibrillator implantation or heart transplant, and thromboembolism. Clot formation is an important complication with an incidence which has been reported to be as high as $50 \%$. The high frequency of thromboembolism is due to the hypercoaguable state of late pregnancy, stasis and turbulent blood flow in dilated cardiomyopathy and the common practice of placing patients in late pregnancy on bed rest.

The outcome for women with PPCM is extremely varied with the serious consequences of the condition usually occurring in the months following the diagnosis. In some patients with PPCM, clinical and echocardiographic status improves quickly and returns to normal. Other patients deteriorate rapidly necessitating device implantation or transplantion. Still others, will respond to therapy and have persistent signs of cardiac dysfunction. US mortality ranges from $25 \%$ to $50 \%$ with close to $50 \%$ of deaths occurring in the first 3 months postpartum.

Women who do recover usually do so within 6 months of the diagnosis and a substantial number of affected women will have significant improvement within the first 6 months. In one study, $50 \%$ of patients with PPCM had resolution of cardiomegaly and LV dysfunction at 6 months after delivery without reported cardiac mortality. Patients with persistent cardiac abnormalities at 6 months after delivery had a mean survival of 4.7 years and an $85 \%$ mortality at 5 years.
Left ventricular size and severity of left ventricular dysfunction are related to worsening outcomes in the acute and subacute phase following the development of PPCM. Poor outcome is associated with worsening LV size and dysfunction, higher parity, being an older patient, and later onset of symptoms following the pregnancy.

Future pregnancies for women with PPCM carry substantial risk. Small studies have shown that women with PPCM have worse outcomes in future pregnancies compared to normal patients even if their left ventricular size and function has returned to normal. Studies have shown worse outcomes for mother and fetus in women with persistent LV dysfunction as compared to women with PPCM who have regained normal function. In one NEJM study, in women with PPCM who regained normal function 25\% of future pregnancies were associated with cardiac dysfunction. In patients with persistent left ventricular dysfunction, 50\% of subsequent pregnancies were associated with worsening cardiac dysfunction. Table 1 shows that women with PPCM had a high incidence of maternal complications during subsequent pregnancy with a higher incidence among those whose LV function had not recovered from the initial pregnancy.

Fetal health has also been found to be worse among women with PPCM in future pregnancies. In the NEJM study, of the women who had regained normal function $13 \%$ of women delivered prematurely. In women with continued left ventricular dysfunction, $50 \%$ of pregnancies ended in premature births and $25 \%$ of the patients required therapeutic abortions.

\section{References}

1. de Beus, et al."Peripartum Cardiomyopathy: a condition intensivists should be aware of.” Intensive Care Medicine. (2003) 29:167-174.

2. Elkayam, etal. "Maternal and Fetal Outcomes of Subsequent Pregnancies in Women with Peripartum Cardiomyopathy.” NEJM. May 24, 2001, Number 21, Volume 344:1567-71.

3. Hibbard, JU, Lindheimer, M, Lang, RM. "A modified definition for peripartum cardiomyopathy and prognosis based on echocardiography." Obstetrics \& Gynecology. 1999;94:311-316.

4. Pearson GD, Veille, JC, Rahimtoola, S, et al "Peripartum cardiomyopathy" National Heart, Lung, and Blood Institute and Office of Rare Diseases (National Institutes of Health) workshop recommendations and review.. JAMA 2000 Mar 1;283(9):1183-8.

5. UptoDate.com

6. Reimold, Sharon, "Peripartum Cardiomyopathy." NEJM. May 24, 2001, Number 21, Volume 344:1629-1630 